Ann. Zootech., I975, 24 (4), 629-638.

\title{
EFFET DE LA TENEUR EN ARGININE DU RÉGIME SUR LA CROISSANCE ET LE BILAN AZOTÉ CHEZ LE LAPIN : RELATION AVEC LE TAUX DE LYSINE
}

\author{
M. COLIN \\ avec la collaboration technique de Marie-Louise Penidon et G. SARdi \\ Station de Recherches sur l'Élevage des Porcs, \\ Centre national de Recherches zootechniques, I. N. R. A., \\ 78350 Jouy en Josas
}

\section{RÉSUMÉ}

Plusieurs auteurs ont récemment signalé que le besoin en arginine du Lapin (o,9 à I,o p. Ioo) paraît très nettement supérieur à celui du Rat ou du Porc $(0,2 \mathrm{p}$. I 00$)$. Mais dans tous ces travaux, le taux en lysine des régimes expérimentaux était très élevé ( $\mathrm{I}, \mathrm{I}$ p. Ioo). On pouvait donc se demander si un antagonisme lysine-arginine comparable à celui mis en évidence chez le Poulet n'existait pas chez le Lapin et n'expliquait pas en fait les résultats obtenus. Nous avons réalisé une expérience nous permettant d'étudier cette hypothèse.

Soixante-six lapins âgés de 5 semaines sont répartis entre les 6 régimes d'un schéma factoriel comprenant 3 taux d'arginine $(0,63-0,78-0,93$ p. Ioo) et deux taux de lysine (o,7 I-I,50 p. Ioo). L'utilisation digestive et métabolique des protéines est mesurée sur 42 de ces animaux. Aux deux taux de lysine, l'addition d'arginine améliore la croissance, l'efficacité alimentaire, le bilan azoté, le coefficient de rétention azotée : ces critères sont optimaux pour 0,93 p. Ioo d'arginine. Dans le cas des régimes apportant 0,78 p. Ioo ou 0,93 p. Ioo d'arginine, ils prennent des valeurs plus élevées à $1,50 \mathrm{p}$. roo de lysine qu'à $0,7 \mathrm{I}$ p. Ioo.

On peut donc en conclure que le besoin en arginine du Lapin se situe bien aux environs de o,9-I,o p. Ioo du régime et qu'il n'a pas été surestimé dans les travaux antérieurs par suite d'un antagonisme lysine-arginine.

Enfin, contrairement à ce que laissaient penser certains résultats rapportés antérieurement, un taux de I,50 p. Ioo de lysine ne paraît pas présenter d'effets dépressifs sur les performances du Lapin.

\section{INTRODUCTION}

Gaman et Fisher (I970) puis Adamson et Fisher (I97I) ont montré que l'arginine est l'un des acides aminés indispensables à la croissance du Lapin. D'autres travaux (McWard, Nicholson et Poulton, i967; Cheeke, I97I ; Addmson et 
FISHER, I973) ont permis d'avancer pour le besoin en arginine du Lapin en croissance des valeurs comprises entre 0,85 et I,OO p. Ioo du régime.

I' après ces données, le Lapin semble donc présenter un besoin en arginine très fortement supérieur à celui du Rat et du Porc (0,2 p. Ioo environ) (N. R. C., I962 ; N. R. C., I973) mais sensiblement de même ordre que celui du Poulet (I,2 p. Ioo) (N. R. C., I97I). Cependant, les trois études sur Lapin précédemment citées ont été réalisées avec des régimes dont les teneurs en lysine sont supérieures à I, I p. Ioo de la ration. Or, quelques expériences indiquent la possibilité d'effets dépressifs de taux de lysine supérieurs à I,o ou à I,2 p. IOo du régime (CHEEKE, I97I; CoL IN, 1973). Par ailleurs, chez le Poulet de nombreux travaux montrent qu'un apport élevé de lysine à certains régimes augmente le besoin en arginine de l'anima1 (JONES, I964; LEWIS, I965; DEAN et SCOTT, I968). Il existe ainsi chez cet Oiseau un antagonisme lysine-arginine (JONES, I964; NESHEIM, I968). Comme l'a rappelé CALET (I972), ce phénomène nécessite de tenir compte de l'équilibre existant dans l'aliment entre la lysine et l'arginine. Il permet en outre d'expliquer que les estimations $\mathrm{du}$ besoin en arginine du Poulet sont beaucoup plus élevées avec des rations à base de caséine (donc très riches en lysine) qu'avec des régimes standard (SNyDER, MorRISON et SCOT'T, I956). On peut alors se demander s'il n'existe pas chez le Lapin des relations de même type entre lysine et arginine. Dans ce cas, les valeurs élevées du besoin, avancées par McWARD, Nicholson et Poulton (I967), CHEEke (I97I), AdAMSON et Fisher (I973), seraient en réalité une conséquence de l'apport trop important de lysine dans les régimes expérimentaux utilisés. Une telle hypothèse peut sembler d'autant plus vraisemblable qu'un antagonisme lysinearginine a été signalé chez un autre Mammifère, le Cobaye (O'DELL et REGAN, I963).

L'expérience dont les résultats sont présentés ici a consisté à étudier une influence éventuelle de la teneur en lysine de la ration sur l'ordre de grandeur du besoin en arginine du Lapin. Nous avons donc envisagé des supplémentations en arginine d'un régime déficient en cet acide aminé et cela à 2 taux de lysine, l'un égal aux recommandations d'apport (CoLIN, I974), l'autre correspondant à une dose susceptible d'entraîner des effets dépressifs par excès (CHEEKE, I97I ; CoLIN, I973).

\section{MATÉRIEL, E'T MÉTHODES}

\section{Présentation de l'expérience}

L'expérience consiste à étudier les performances de croissance et d'efficacité alimentaire de 66 lapins répartis entre les 6 régimes d'un schéma factoriel comprenant 2 taux de lysine (o,7 I et I,50 p. 100 du régime) et 3 taux d'arginine $(0,63-0,78-0,93$ p. 100). De plus, pour 42 des animaux (7 par régime), nous pratiquons un bilan azoté par la méthode des collectes selon des modalités déjà décrites (Colis, I974).

\section{Animaux.}

Les 66 lapins de race californienne ou croisés (LEBAs, I975) sont âgés de 35 ou 36 jours en début d'expérience et pèsent alors $890 \mathrm{~g}$ en moyenne. Les bilans azotés sont établis en plaçant individuellement 42 des lapins dans des cages déjả décrites (Colis, I974). Les autres sont logés dans des cages individuelles entièrement métalliques et munies d'abreuvoirs automatiques à surface d'eau libre. Les répétitions sont constituées de lapins ayant le même poids à + et 5 semaines d'âge. 


\section{Régimes.}

Les rations expérimentales sont principalement à base de levures de distillerie, de luzerne déshydratée et de maïs. L'association de ces matières premières selon les proportions rapportées au tableau I permet en effet de disposer d'un régime de base pauvre en arginine et correctement équilibré en lysine d'après des résultats antérieurs (CoLIN, 1974). Un apport de DL-méthionine $(0,2$ p. Ioo) évite une carence en acides aminés soufrés. La L-lysine monochlorhydrate ou la L-arginine chlorhydrate sont ajoutées en substitution, sur une base isoazotée, d'une partie du glutamate de sodium du régime non supplémenté. Les teneurs en chlore et en sodium des régimes sont approximativement égalisées par des apports différentiels de chlorure de sodium et de bicarbonate de sodium.

TABLEAU I

Composition des régimes expérimentaux

\begin{tabular}{|c|c|c|c|c|c|c|}
\hline $\begin{array}{l}\text { Teneur en lysine } \\
\text { p. } 100 \text { du régime }(\mathbf{1})\end{array}$ & \multicolumn{3}{|c|}{0,71} & \multicolumn{3}{|c|}{1,50} \\
\hline $\begin{array}{l}\text { Teneur en arginine } \\
\text { p. } \mathbf{1 0 0} \text { du régime }\left({ }^{1}\right)\end{array}$ & 0,63 & 0,78 & 0,93 & 0,63 & 0,78 & 0,93 \\
\hline Luzerne $\left({ }^{2}\right)$ & 30 & 30 & 30 & 30 & 30 & 30 \\
\hline Levure $\left(^{3}\right) \ldots$ & 10 & 10 & 10 & 10 & 10 & 10 \\
\hline Maïs $\left.{ }^{4}\right) \ldots \ldots \ldots \ldots \ldots \ldots \ldots$ & 40 & 40 & 40 & 40 & 40 & 40 \\
\hline Paille d'orge $\ldots \ldots \ldots \ldots \ldots \ldots$ & 5 & 5 & 5 & 5 & 5 & 5 \\
\hline DL-Méthionine $\ldots \ldots \ldots \ldots \ldots$ & 0,2 & 0,2 & 0,2 & 0,2 & 0,2 & 0,2 \\
\hline Chlorbydrate de L-lysine $\left(^{5}\right) \ldots$ & - & - & - & 1,05 & 1,05 & 1,05 \\
\hline L-arginine $\mathrm{HCl}\left({ }^{6}\right) \ldots \ldots \ldots \ldots$ & 0 & 0,18 & 0,36 & 0 & 0,18 & 0,36 \\
\hline Glutamate de sodium $\ldots \ldots \ldots$ & 3,5 & 2,90 & 2,3 & 1,7 & 1,1 & 0,5 \\
\hline Chlorure de sodium . ......... & 0,55 & 0,50 & 0,45 & 0,27 & 0,22 & 0,17 \\
\hline Bicarbonate de sodium $\ldots \ldots \ldots$ & 0 & 0,18 & 0,37 & 0,62 & 0,80 & 0,93 \\
\hline $\begin{array}{c}\text { Complément minéral et vitami- } \\
\text { nique }\left({ }^{7}\right) \ldots \ldots \ldots \ldots \ldots \ldots\end{array}$ & 2 & 2 & 2 & 2 & 2 & 2 \\
\hline Amidon de maïs...$\ldots \ldots \ldots$ & 8,75 & 9,04 & 9,32 & 9,16 & 9,45 & 9,74 \\
\hline Résultats d'analyse & & & & & & \\
\hline Matière sèche............. & 90,5 & 90,9 & 90,3 & 90,4 & 90,2 & 90,4 \\
\hline Matière organique $\ldots \ldots \ldots \ldots$ & 83,8 & 83,5 & 83,6 & 83,9 & 83,9 & 83,4 \\
\hline Matière azotée $(\mathrm{N} \times 6,25) \ldots \ldots$ & 16,5 & 16,8 & 16,4 & 15,8 & 16,1 & 16,6 \\
\hline$\ldots \ldots \ldots \ldots \ldots$ & 10,6 & $10,{ }^{\prime}$ & 10,0 & 10,2 & 10,6 & 10,7 \\
\hline
\end{tabular}

(1) Valeurs calculées d'après les tables de composition en acides aminés (PIon, 1973).

( $\left.{ }^{2}\right) 91$ p. 100 de matière sèche ; 14,3 p. 100 de matières azotées $(\mathrm{N} \times 6,25)$.

(3) 90,1 p. 100 de matière sèche; 51,2 p. 100 de matières azotées $(\mathrm{N} \times 6,25)$.

(4) 87,1 p. 100 de matière sèche; 10,2 p. 100 de matières azotées $(\mathrm{N} \times 6,25)$.

(5) Commercialisée par Ugine Kulhmann. 95 p. 100 d'hydrochlorate de L-lysine à 80 p. 100 de lysine de base.

$\left({ }^{6}\right)$ Commercialisée par Vegetadrog. 99 p. 100 de L-arginine chlorhydrate à 82 p. 100 d'arginine de base.

(7) Composition minéraux en p. $100 \mathrm{~d} u$ CMV : carbonate de magnésium 24,0; phosphate bicalcique 74,5 ; iodure de potassium 0,008 ; sulfate de cuivre 0,060 ; sulfate de zinc 0,200 ; sulfate de cobalt 0,120 ; sulfate de manganèse 0,120 ; sulfate de fer 0,992 vitamines : Colin (1975).

La valeur énergétique mesurée des régimes est de 3830 kcal pour énergie brute et de 27 Io pour l'énergie digestible. Leur taux protéique est égal à $\mathbf{1 6 , 3}+0,2 \mathrm{p}$. Ioo du régime. I.es aliments sont présentés sous forme de granulés de $5 \mathrm{~mm}$ de diamètre. 
Contrôles des performances.

Les performances de croissance sont contrôlées pendant 5 semaines, c'est-à-dire la majeure partie de la période comprise entre le sevrage (4 semaines) et l'abattage (Io-I I semaines).

Les animaux sont pesés individuellement une fois par semaine vers $9 \mathrm{~h}$ du matin. Les consommations individuelles sont mesurées 2 fois par semaine par différence entre les quantités d'aliments distribuées et refusées.

La période pendant laquelle on établit le bilan azoté pour une partie des animaux dure io jours et débute 8 jours après le commencement de l'expérience.

\section{Analyse statistique.}

L'expérience est menée selon un schéma factoriel en bloc complet équilibré. L'exploitation statistique des résultats est assurée par analyse de variance et par un test de NEwmanN et KEULs (DAGNELIE, I970).

\section{RÉSULTATS}

\section{A. - Croissance et efficacité alimentaire}

La croissance pondérale des animaux est améliorée par l'addition de lysine et d'arginine au régime de base (tab1. 2). Ces deux effets sont statistiquement hautement significatifs. Globalement, l'apport de 0,15 ou de 0,30 p. Ioo d'arginine aux aliments

TABLEAU 2

Résultats moyens de croissance et de consommation obtenus sur l'ensemble des animaux

\begin{tabular}{|c|c|c|c|c|c|c|c|c|c|}
\hline $\begin{array}{l}\text { Teneur en lysine } \\
\text { p. } 100 \text { du régime }\end{array}$ & \multicolumn{3}{|c|}{0,71} & \multicolumn{3}{|c|}{1,50} & \multirow{2}{*}{\multicolumn{3}{|c|}{$\begin{array}{l}\text { Signification } \\
\text { statistique }\left({ }^{3}\right)\end{array}$}} \\
\hline $\begin{array}{l}\text { Teneur en arginine } \\
\text { p. } 100 \text { du régime }\end{array}$ & 0,63 & 0,78 & 0,93 & 0,63 & 0,78 & 0,93 & & & \\
\hline $\begin{array}{l}\text { Poids en début } \\
\text { d'expérience }(g)\end{array}$ & $\begin{array}{r}888 \\
\pm \quad 2 /\end{array}$ & $\begin{array}{r}894 \\
\pm \quad 24\end{array}$ & $\begin{array}{r}890 \\
\pm 28\end{array}$ & $\begin{array}{r}898 \\
+\quad 29\end{array}$ & $\begin{array}{r}898 \\
\pm \quad 24\end{array}$ & $\begin{array}{r}895 \\
+\quad 29\end{array}$ & & & \\
\hline Gain de poids (g/j) & $\begin{array}{c}32,7^{a} \\
\pm 1,0(1)\end{array}$ & $\begin{array}{r}32,8^{a} \\
\pm \quad 1,3\end{array}$ & $\begin{array}{r}35,4^{a b} \\
\pm 1,1\left({ }^{2}\right)\end{array}$ & $\begin{array}{l}33,2 a b \\
\pm 1,2\end{array}$ & $\begin{array}{r}36,2^{a b c} \\
+\quad 1,0\end{array}$ & $\begin{array}{r}38,2^{c} \\
+\quad 1,6\end{array}$ & $\begin{array}{c}\text { A } \\
\text { L } \\
\text { I }\end{array}$ & $\begin{array}{l}7,03 \\
8,97 \\
2,56\end{array}$ & $\begin{array}{l}* * \\
* * \\
\text { NS }\end{array}$ \\
\hline Consommation $(g / j)$ & $\begin{array}{l}100^{\alpha} \\
\pm 3\end{array}$ & $\begin{array}{l}10^{\prime a b} \\
\pm 5\end{array}$ & $\begin{array}{l}103^{a b} \\
\pm 3\end{array}$ & $\begin{aligned} & 99^{\alpha} \\
\pm & 3\end{aligned}$ & $\begin{array}{l}110^{b} \\
\pm 3\end{array}$ & $\begin{array}{l}107^{a b} \\
\pm 4\end{array}$ & $\begin{array}{c}\text { A } \\
\text { L. } \\
\text { I }\end{array}$ & $\begin{array}{r}3,78 \\
<1 \\
1,55\end{array}$ & $\begin{array}{l}* * \\
\text { NS } \\
\text { NS }\end{array}$ \\
\hline $\begin{array}{c}\text { Indice de } \\
\text { consommation }\end{array}$ & $\begin{aligned} & 3,04^{a b} \\
\pm & 0,06\end{aligned}$ & $\begin{aligned} & 3,19^{a} \\
\pm & 0,11\end{aligned}$ & $\begin{array}{ll} & 2,92^{b c} \\
\pm & 0,08\end{array} \mid$ & $\begin{array}{ll} & 2,98^{b c} \\
\pm & 0,09\end{array}$ & $\begin{aligned} & 3,06 a b \\
\pm & 0,06\end{aligned}$ & $\begin{aligned} & 2,82 c \\
\pm & 0,09\end{aligned}$ & $\begin{array}{c}\text { A } \\
\text { L } \\
\text { I }\end{array}$ & $\begin{array}{l}4,63 \\
1,91 \\
1,36\end{array}$ & $\begin{array}{l}* \\
\text { NS } \\
\text { NS }\end{array}$ \\
\hline
\end{tabular}

(1) Écart-type de la moyenne.

(2) $a, b, c$, les données ayant en indice une lettre différente, diffèrent significativement au seuil $\mathrm{P}=0,05$.

(3) NS : non significatif; $*$ : seuil 0,$05 ; * *$ : seuil 0,$01 ; * * *$ : seuil 0,001 .

A : effet arginine; L : effet lysine; I : effet interaction. 
non supplémentés augmente significativement la croissance des lapins. Cependant l'addition de $0,30 \mathrm{p}$. Ioo d'arginine au régime de base provoque un accroissement du gain de poids plus important pour les régimes à $I, 50$ p. Ioo de lysine ( + I 5 p. roo) que pour ceux à $0,7 \mathrm{I}$ p. Ioo de lysine $(+8 \mathrm{p}$. Ioo). Pareillement, l'augmentation du taux de lysine de $0,7 \mathrm{I}$ à $\mathrm{I}, 5^{\circ} \mathrm{p}$. Ioo du régime ne modifie les performances pondérales que pour les animaux recevant des rations à 0,78 ou $0,93 \mathrm{p}$. Ioo d'arginine.

\section{TABLEAU 3}

Résultats moyens de digestibilité et de bilan azoté obtenus sur 42 animaux

\begin{tabular}{|c|c|c|c|c|c|c|c|c|c|}
\hline $\begin{array}{l}\text { Teneur en lysine } \\
\text { p. } 100 \text { du régime }\end{array}$ & & 0,71 & & \multicolumn{3}{|c|}{1,50} & \multirow{2}{*}{\multicolumn{3}{|c|}{$\begin{array}{l}\text { Signification } \\
\text { statistique }(\mathbf{1})\end{array}$}} \\
\hline $\begin{array}{l}\text { Tenear en arginine } \\
\text { p. } 100 \text { du régime }\end{array}$ & 0,63 & $0,7 x$ & 0,93 & 0,033 & 0,78 & $0,9: 3$ & & & \\
\hline Gain cle poids $(\mathrm{g} / \mathrm{j})$ & $\begin{array}{r}35,9^{a} \\
\pm 2,3(\mathbf{1})\end{array}$ & $\begin{aligned} & 33,1^{a} \\
\pm & 1,8\end{aligned}$ & $\begin{array}{r}\quad 0,7 a b \\
+\quad 1,7(1)\end{array}$ & $\begin{aligned} & 37,33^{a b} \\
\pm & 2,1\end{aligned}$ & $\begin{aligned} & 22,0 a b \\
\pm & 2,5\end{aligned}$ & $\begin{array}{r}46,1^{b} \\
\pm \quad 3,1\end{array}$ & $\begin{array}{c}\text { A } \\
\text { L } \\
\text { I }\end{array}$ & $\begin{array}{l}9,59 \\
7,25 \\
1,1\end{array}$ & $\begin{array}{l}* * * \\
* * \\
\text { NS }\end{array}$ \\
\hline CUD Matière sèche $\left({ }^{2}\right)$ & $\begin{array}{r}73,8 \\
\pm 0,8\end{array}$ & $\begin{array}{r}73,9 \\
+\quad 1,2\end{array}$ & $\begin{array}{r}73,2 \\
\pm \quad 1,2\end{array}$ & $\begin{array}{r}73,5 \\
\pm \quad 1,2\end{array}$ & $\begin{array}{r}73,2 \\
\pm \quad 0,6\end{array}$ & $\begin{array}{r}73,7 \\
\pm 1,2\end{array}$ & $\begin{array}{l}\text { A } \\
\text { L } \\
\text { I }\end{array}$ & $\begin{array}{l}<1 \\
<1 \\
<1\end{array}$ & $\begin{array}{l}\text { NS } \\
\text { NS } \\
\text { NS }\end{array}$ \\
\hline $\begin{array}{l}\text { CUD Matière } \\
\text { organique }\left({ }^{2}\right)\end{array}$ & $\begin{array}{r}74,{ }_{1}^{\prime} \\
\pm 0,9\end{array}$ & $\begin{array}{r}74,5 \\
\pm 1,2\end{array}$ & $\begin{array}{r}73,8 \\
+\quad 1,2\end{array}$ & $\begin{array}{r}7 \%, 1 \\
+\quad 1,3\end{array}$ & $\begin{array}{r}74,0 \\
+\quad 0,6\end{array}$ & $\begin{array}{r}74,0 \\
\pm \quad 1,2\end{array}$ & $\begin{array}{c}\text { A } \\
\text { L } \\
\text { I }\end{array}$ & $\begin{array}{l}<1 \\
<1 \\
<1\end{array}$ & $\begin{array}{l}\text { NS } \\
\text { NS } \\
\text { NS }\end{array}$ \\
\hline CU1) Azote $\left({ }^{2}\right)$ & $\begin{array}{r}77,0 \\
\pm 1,3\end{array}$ & $\begin{array}{r}75,5 \\
+\quad 2,3\end{array}$ & $\begin{array}{r}71,6 \\
\pm \quad 1,6\end{array}$ & $\begin{array}{r}75,3 \\
+\quad 2,2\end{array}$ & $\begin{array}{r}73,7 \\
\pm 1,1\end{array}$ & $\begin{array}{r}75,4 \\
\pm 1,7\end{array}$ & $\begin{array}{c}\text { A } \\
\text { L } \\
\text { I }\end{array}$ & $\begin{array}{l}<1 \\
<1 \\
<1\end{array}$ & $\begin{array}{l}\text { NS } \\
\text { NS } \\
\text { NS }\end{array}$ \\
\hline CR Azote $\left({ }^{2}\right)$ & $\begin{array}{r}72,8^{a} \\
\pm 1,4^{a}\end{array}$ & $\begin{aligned} & 71,6^{a} \\
&+\quad 2,0\end{aligned}$ & $\begin{array}{l}77,4 b c \\
\pm 1,4\end{array}$ & $\begin{array}{l}75,2 a b \\
\pm 1,8\end{array}$ & $\begin{array}{l}7: 3,1 a b \\
\pm 1,4\end{array}$ & $\begin{array}{r}80,4^{c} \\
\pm \quad 1,4\end{array}$ & $\begin{array}{c}\text { A } \\
\text { L } \\
\text { I }\end{array}$ & $\begin{array}{l}8,71 \\
2,9 \prime \\
<1\end{array}$ & $\begin{array}{l}* * \\
\text { NS } \\
\text { NS }\end{array}$ \\
\hline Bilan azotć $(\mathrm{g} / \mathrm{j})\left({ }^{2}\right)$ & $\begin{aligned} & 1,3^{a b} \\
\pm & 0,08\end{aligned}$ & $\begin{aligned} & 1,3,3^{a} \\
\pm & 0,06\end{aligned}$ & $\begin{array}{l}1,52^{6} \\
\pm 1,07\end{array}$ & $\begin{array}{l}1,3 a \\
+\quad 0,11\end{array}$ & $\begin{aligned} & 1.51^{b} \\
+ & 0,06\end{aligned}$ & $\begin{array}{r}1,75^{c} \\
\pm 0,00\end{array}$ & $\begin{array}{c}\text { A } \\
\text { L } \\
\text { I }\end{array}$ & $\begin{array}{l}5,26 \\
2,59 \\
2,27\end{array}$ & $\begin{array}{l}* \\
\text { NS } \\
\text { NS }\end{array}$ \\
\hline CUP Azote & $\begin{array}{r}56,1 \\
+1,5\end{array}$ & $\begin{array}{r}53,8 \\
+\quad 1,9\end{array}$ & $\begin{array}{r}57,7 \\
+1,6\end{array}$ & $\begin{array}{r}56,7 \\
\pm \quad 2,1\end{array}$ & $\begin{array}{r}53,9 \\
\pm \quad 1,6\end{array}$ & $\begin{array}{r}61,2 \\
\pm 2,1\end{array}$ & $\begin{array}{c}\text { A } \\
\text { L } \\
\text { I }\end{array}$ & $\begin{array}{l}1,51 \\
<1 \\
<1\end{array}$ & $\begin{array}{l}\text { NS } \\
\text { NS } \\
\text { NS }\end{array}$ \\
\hline
\end{tabular}

(1) Voir tableau 1 .

$\left.{ }^{2}\right) \quad$ CUD $=\frac{\text { Élément ingéré }- \text { Élément excrété par les fèces }}{\text { Élément ingéré }} \times 100$.

CR azote $=\frac{\text { Azote ingéré }- \text { Azote excrété clans les fèces et l'urine }}{\text { Azote ingéré }- \text { Azote excrété dans les fèces }} \times 100$.

CUP azote $=\frac{\text { Azote ingéré }- \text { Azote excrété dans l'urine et les fèces }}{\text { Azote ingéré }} \times 100$. 
Il semble donc exister une interaction entre la teneur en lysine de la ration et le niveau de supplémentation en arginine. Cette interaction est d'ailleurs à la limite de la signification statistique $(\mathrm{P}<\mathrm{O}, \mathrm{I})$.

On observe également une augmentation significative de la consommation lors de l'addition d'arginine. L'effet arginine est statistiquement significatif et oppose les régimes à 0,78 et 0,93 p. Ioo d'arginine aux deux régimes non supplémentés (tabl. 2). Mais une fois encore, les effets de l'apport d'arginine sont plus marqués pour I,50 p. Ioo de lysine que pour $0,7 \mathrm{I}$ p. Ioo. Par contre, l'addition de 0,79 p. Ioo de lysine n'augmente pas significativement les quantités ingérées.

Les indices de consommation obtenus avec les deux régimes à $0,78 \mathrm{p}$. Ioo d'arginine sont significativement plus élevés que ceux enregistrés avec les régimes à 0,93 p. Ioo d'arginine (tabl. 2). A l'inverse, les différences observées pour l'indice de consommation entre les deux taux de lysine sont non significatives.

\section{B. - Digestibilité}

Les variations des taux de lysine ou d'arginine dans les régimes ne paraissent pas affecter les coefficients d'utilisation digestive apparente de la matière sèche, de la matière organique ou de l'azote (tabl. 3).

\section{C. - Bilan azoté et utilisation métabolique de l'azote}

Le bilan azoté varie de façon parallèle au gain de poids : il est significativement amélioré par l'addition d'arginine et cette augmentation est plus forte pour les régimes à I,50 p. Ioo de lysine (+30 p. Ioo) que pour ceux à $0,7 \mathrm{I}$ p. Ioo de lysine ( + I7 p. IOO). La comparaison des bilans azotés obtenus avec des régimes à $0,7 \mathrm{I}$ et $\mathrm{I}, 50 \mathrm{p}$. Ioo de lysine ne permet pas d'observer des écarts importants lorsque le taux d'arginine est de 0,63 p. Ioo. On enregistre par contre des différences non négligeables en faveur des régimes à $\mathrm{I}, 5^{\circ} \mathrm{p}$. Ioo de lysine lorsque le taux d'arginine est de 0,78 p. Ioo ( + I3,5 p. I00) et 0,93 p. IOo ( + I5 p. IOO). Le coefficient de rétention azotée présente une évolution comparable au bilan azoté. Le coefficient d'utilisation pratique de l'azote varie peu. Il présente toutefois les valeurs les plus élevées pour $0,93 \mathrm{p}$. Ioo d'arginine dans le régime et ne paraît pratiquement pas modifié par la supplémentation en lysine.

\section{DISCUSSION}

\section{A. - Effets de l'addition de lysine}

Dans cette expérience, un taux élevé de lysine a permis des performances égales ou sensiblement supérieures à celles obtenues avec des teneurs de lysine correspondant aux recommandations établies antérieurement (CoLIN, I974; I975) Or les conséquences de l'incorporation de doses importantes de lysine au régime sont très discutées. ChEekE (I97I), Colin (I973) signalent des possibilités de déséquilibres par excès lorsque le taux de lysine dépasse I,o p. Ioo du régime. Par contre, 
Chenke et Amberg (I972), Anonyme (I973) ne trouvent dans ces conditions aucune toxicité. Nous n'avons nous-mêmes enregistré aucun effet dépressif en ajoutant des doses croissantes de lysine (jusque I,40 p. Ioo) à des régimes du même type que ceux utilisés dans l'expérience rapportée ici (CoL,IN, données non publiées). En fait, il n'est pas impossible que les effets dépressifs observés précédemment (CHEEkE, I97 I CoLIN, I973) soient partiellement dus à un déséquilibre minéral. Dans ces travaux, la lysine est en effet introduite dans les rations sous forme de chlorhydrate et se trouve substituée à du glutamate de sodium. On modifie donc le rapport entre sodium et chlore. Ainsi, dans 1'expérience de CoLIN (I973), lors de 1'addition de la dose la plus élevée de lysine, le taux de sodium est abaissé de 0,33 p. Ioo à 0,25 p. Ioo tandis que celui de chlore est élevé de 0,37 à 0,48 p. Ioo đu régime. Chez le Poulet, une telle variation des taux de chlore et de sodium est susceptible de déprimer de façon non négligeable les performances pondérales des animaux (MONGIN et SAUvEUR, 1973). Si des phénomènes comparables existent chez le Lapin, une partie des effets dépressifs observés lors de l'addition de chlorhydrate de lysine peut en réalité être due à la modification du rapport $\mathrm{Na} / \mathrm{Cl}$. Cette hypothèse rend également compte du fait que dans l'expérience rapportée ici, l'addition d'une quantité importante de lysine purifiée ne déprime par les performances des lapins puisque les taux de chlore et de sodium sont maintenus constants d'un régime à l'autre.

Par ailleurs, il faut remarquer que l'addition de lysine n'augmente sensiblement le gain de poids et l'efficacité alimentaire que lorsque les régimes sont supplémentés en arginine. Il est vraisemblable que l'arginine constitue le facteur limitant primaire du régime de base. L'addition d'arginine supprimant cette déficience permet la mise en évidence d'une action amélioratrice de l'apport de lysine supplémentaire. La lysine paraît donc être le facteur limitant secondaire du régime de base. Dans les aliments du type de ceux utilisés ici, un apport de o,7I p. Ioo de lysine ne couvre donc pas la totalité du besoin en lysine du Lapin. En fait, des travaux antérieurs (CoLIN, I974) ont déjà montré que si 0,65 p. roo de lysine dans la ration suffit pour obtenir des performances de bon niveau, le besoin en lysine au sens strict du terme semble plus élevé. Par exemple, un régime à 2 I p. Ioo de protéines et 0,80 p. Ioo de lysine est encore amélioré par addition de $0,3 \mathrm{I}$ p. Ioo de lysine. Des phénomènes comparables se produisent vraisemblablement dans l'expérience présentée ici. En outre, il est possible qu'une fraction non négligeable de la lysine du régime de base soit indisponible. En effet, 25 p. Ioo environ de la lysine sont apportés par les protéines de luzerne déshydratée qui ont subi des traitements thermiques. Or, ceux-ci entraînent souvent des réactions de Maillard qui rendent indisponible une partie de la lysine (Godon et PeTtT, I97 I ; LEBAs, Dolz et Espaignet, r973). La taux de lysine disponible peut donc être dans ces régimes sensiblement inférieur aux recommandations précédemment établies (CoI,IN, I974). Ce phénomène s'il existe expliquerait également l'amélioration des performances par apport de lysine supplémentaire.

\section{B. - Effets de l'addition d'arginine}

On observe aux deux taux de lysine une amélioration sensible des performances lors de l'addition d'arginine aux régimes de base. Les meilleurs résultats sont enregistrés pour 0,93 p. Ioo d'arginine. Dans le cas des rations apportant $1,50 \mathrm{p}$. Ioo de lysine, les croissances des lapins augmentent de façon pratiquement proportionnelle 
au taux d'arginine de l'aliment. Par contre, à 0,7 I p. Ioo de lysine on n'enregistre pas de différences entre les gains pondéraux des lapins correspondant aux régimes à 0,63 et 0,78 p. Ioo d'arginine. Cette similitude des résultats obtenus est vraisemblablement due à des problèmes d'échantillonnage et de variabilité individuelle plutôt qu'à une identité nutritionnelle de ces 2 aliments.

L'étude des résultats obtenus pour le bilan azoté montre que celui-ci évolue de façon sensiblement parallèle au gain de poids. L'amélioration de la croissance par addition d'arginine est bien due à une augmentation de la protéinogenèse, ce que confirme d'ailleurs l'évolution des coefficients de rétention azotée d'un régime à l'autre. Par ailleurs, on n'observe aucune influcnce des supplémentations sur les différents coefficients d'utilisation digestive apparente. Tous ces faits concernant l'évolution de la digestibilité, du bilan azoté et de l'utilisation métabolique de l'azote lors d'une supplémentation en arginine sont en accord avec les observations effectuées antérieurement pour la méthionine (CoLIN et ARkhURst, I973) et la lysine (Colin, I974).

Le Lapin présenterait donc, d’après les résultats observés ici un besoin élevé en arginine, égal ou supérieur à 0,93 p. Ioo de la ration. Il paraît cependant illusoire d'avancer une valeur précise du besoin. Fin effet, il n'a pas été possible parmi les traitements étudiés de mettre en évidence un taux d'arginine à partir duquel l'addition de nouvelles doses de cet acide aminé au régime ne modifie plus les performances. Le besoin peut donc être sensiblement supérieur à 0,93 p. Ioo. Par ailleurs, on ne dispose pratiquement pas d'informations sur la disponibilité de l'arginine dans les protéines des matières premières utilisées.

L'obtention chez le Lapin de croissances et de bilans azotés de haut niveau est conditionnée, aux deux taux de lysine étudiés par une teneur en arginine du régime au moins égale à 0,93 p. IoO.

Cette dose est très proche des estimations du besoin en arginine du Lapin formulées par McWard, Nicholson et Poulton en ig67 (o,98 p. IOo) ; CheEke, en I97I (o,88 p. IOO), ainsi que par ADAMSON et FishER en I973 ( I,Oo p. IOO). Cette expérience permet donc d'affirmer que les résultats des auteurs précédents n'ont pas été faussés par le taux élevé de lysine des aliments qu'ils ont utilisés.

Le Lapin présente bien un besoin en arginine très fortement supérieur à celui du Rat ou du Porc (NRC I962; NRC I973) et du même ordre de grandeur que celui du Poulet (NRC I97I). Cette caractéristique du Lapin déjà signalée par CHEEKE (I97I) ne semble pas dépendre du taux de lysine. Elle ne serait donc pas la conséquence, comme nous en avons émis l'hypothèse, d'un antagonisme lysine-arginine qui aurait considérablement augmenté le besoin en arginine du Lapin dans les régimes utilisés par McWard, Nicholson et Poulton (I967), Chenke (I97I), Adamson et Fisher (I973).

\section{CONCIUSION}

En conclusion, ce travail confirme l'existence d'un besoin en arginine élevé chez le Lapin en croissance, égal ou supérieur à 0,9 p. Ioo du régime. Ce résultat est en accord avec les données bibliographiques sur ce sujet (McWARD, Nicholson et Pouliton, I967; ChEEke, I97I, AdAMson et Fisher, I973). Mais surtout, cette 
étude tend à infirmer l'hypothèse qu'un antagonisme lysine-arginine pourrait entraîner une forte augmentation du besoin en arginine dans le cas de régimes riches en lysine. Enfin, il ne semble pas que dans les conditions de cette expérience, des doses importantes de lysine (de l'ordre de I,5 p. Ioo) présentent chez le Lapin un effet dépressif.

Regu pour publication en avril 1975.

\title{
SUMMARY
}

\author{
EFFECT OF THE DIETARY ARGININE CONTENT ON GROWTH \\ AND NITROGEN BALANCE IN THE RABBIT : \\ RELATIONSHIP WITH THE LYSINE LEVEI,
}

Recent data of several authors indicate that the requirement for arginine of the rabbit ( 0.9 to I.o p. Ioo) seems to be definitely higher than that of the rat or the pig (0.2 p. Ioo). However, in all these studies, the lysine level of the experimental diets was very high (I.I p. roo). Therefore, it may be asked whether a lysine-arginine antagonism comparable to that evidenced in the chicken also exists in the rabbit, thus explaining the results obtained. In order to verify this hypothesis, we carried out the following experiment:

Sixty-six 5-weeks-old rabbits were alloted to 6 treatments of a factorial design including 3 arginine levels (0.63-0.78-0.93 p. I0o) and 2 lysine levels (0.7I-I.5O p. IOO). The apparent digestibility and metabolic utilization of protein were measured on 42 of these animals. For the two lysine levels, addition of arginine improved growth, feed efficiency, nitrogen balance, nitrogen retention coefficient the highest values being obtained with $0.93 \mathrm{p}$. Ioo arginine. In the case of diets supplying 0.78 or 0.93 p. Ioo arginine, the values obtained were higher with I.5o than with $0.7 \mathrm{I}$ p. roo lysine.

It may be concluded that the requirement for arginine in the rabbit is effectively located around 0.9-I.o p. Ioo of the diet and that it has not been overestimated in previous works because of a lysine-arginine antagonism.

Contrarily to what could be expected from some already reported results, a lysine level of $\mathrm{I} .50 \mathrm{p}$. roo does not seem to exert any depressive effect on rabbit performances.

\section{RÉFÉRENCES BIBLIOGRAPHIQUES}

Adamson I., Fisher H., r97 t. The aminoacid requirement of the growing rabbit : qualitative needs. Nutp. Repts Int., 4, 59-64.

Adamson I., Fisher H., I973. The aminoacid requirement of the growing rabbit : an estimate of quantitative needs. J. Nutr., 103, I306-r 310.

Anonyme, I973. Alimentation de la volaille et d'autre petit bétail II lapins. Centre de Recherches agronomiques de l'Etat de Gand. Rapport d'acitvité I973, I4I-I45.

CaLeT C.,I972. Acquisitions récentes en alimentation des volailles. Deuxième congrès mondial de l'alimentation animale, Madrid, $23-28$ octobre 1972 , 157-218. Carsi éd.

Chezke P. R., 1971. Arginine, lysine and Methionine needs of the growing rabbit. Nutr. Repts. Int., 3, I23-I28.

Cheeke P. R., Amberg J. W., :972. Protein nutrition of the rabbit. Nutr. Repts. Int., 5, $259-266$.

Colin M., i973. Influence de l'addition de lysine à des régimes à base de tourteau de sésame chez le Lapin. Journées Recherches Avicoles et Cunicoles, 19-2 I, I. T. A. V. I. éd.

Colin M., I974. Supplémentation en Iysine d'un régime à base de tourteau de sésame chez le Lapin. Effets sur les performances de croissance et le bilan azoté estimé par deux méthodes. Ann. Zootech., 23, I I 9-132.

Colin M., I975. Effets sur la croissance du Lapin de la supplémentation en L-lysine et DL-méthionine de régimes végétaux simplifiés. Ann. Zootech., 24, 465-474. 
Colin M., Arkiurst G., I973. Fiffets de l'addition de IDI-méthionine à un régime à base de tourteau de soja sur la croissance et la rétention azotée chez le lapin. Journées Recherches aricoles cunicoles, I9-2I, I. T. A. V. 1, éd.

I) AGNeLif: P., 1970. Théories et méthodes statistiques, vol, 2, ied. Duculot, S. A. (iembloux.

DEAN W. F., ScotT H. M., I 968 . Ability of arginine to reverse the growth depression induced by supplementing a crystalline aminoacid diet with excess of lysine. Poultry Sci., 40, 34I-342.

Gaman E., Fisher H., I97x. The essentiality of arginine, lysine and methionine for the growing rabbit. Nutr. Repts. Int., 1, 57-64.

Godon B., Petit L., I97I. Le maïs grain : préstockage, stockage et qualité. V Propriété des protéines. Ann. Zootech., 20, h.s., 64I-644.

Jones J. D., I964. Lysine-arginine antagonism in the chick. J. Nutr., 84, 3I3-32I.

Lebas F, Dolz J, Espaignet A., 1973. Influence des conditions de séchage de la luzerne déshydratée sur les performances de croissance de lapins recevant une alimentation à base de luzerne. Journées Recherches avicoles cunicoles. I5-I6, I. T. A. V. I., éd.

LEBAS F., I975. Influence de la teneur en énergie de l'aliment sur les performances de croissance chez le Lapin. Ann. Zootech., 24, 28I-288.

Lewis D., 1965. The concept of agent and target in amino-acid interactions. Proc. Nutr. Soc., 24, I96-202.

McWard G. W., Nicholson L. B., Poulton B. R., I967. Arginine requirement of the young rabbit. J. Nutr., 92, I $18-120$.

Mongin P., Sauveur B., I973. Effets des teneurs en chlore, sodium et potassium du régime sur la croissance du Poulet et l'apparition des anomalies cartilagineuses. Journées Recherches Avicoles et cunicoles, I87-192, I. T. A. V. I. éd.

National Research Council Committee on Animal Nutrition, 1962. Nutrient requirements for laboratory animals, publ. 990. National Academy of Science Washington, D. C.

National Research Council Committee on Animal Nutrition, ig7i. Nutrient Requirements of Poultry, Sixth revised edition. National Academy of Science Washington, D. C.

National Research Council Committee on Animal Nutrition, 1973. Nutrient Requirements of swine, National Academy of Sciences Washington. D. C.

Nesherm M. C., I969. Kidney arginase activity and lysine tolerance in strains of chickens selected for high or low requirement of arginine. J. Nutr., 95, 79-87.

O'DeLI B. L., REGAN W. O., I963. Effect of lysine and glycine open arginine requirement of the guineapig. Proc. Soc. Exp. Biol. Med., 112, 336-337.

Pion R., I973. Composition en acides aminés des aliments azotés. Facteurs agronomiques de variation. Journées d'Information sur l'Alimentation azotée des animaux. Paris $2 \mathbf{1}-22$ novemtre 1973 I. N. R. A. éd., II-I4.

Snyder J. M., Morrison W. D., Scott H. M., 1956. The arginine requirement of chicks fed purified and corn-soya diets. Poulty sci., 35, $852-855$. 